\title{
Large-area synthesis of carbon nanofibres at room temperature
}

Bojan 0. Boskovic, Vlad Stolojan, Rizwan U.A. Khan, Sajad Haq and S. Ravi P. Silva

Nature Materials 1, 165-168 (2002).

In this letter, on page 166, column one, line two, and page 167, column two, line 11, the units of pressure were incorrectly stated as 1,000 mbar. This should read 1,000 mTorr in both cases. 